\title{
Finite element study of the effect of some local defects on the risk of transverse cracking in continuous casting of steel slabs
}

\author{
F. Pascon, A.M. Habraken \\ Department of Mechanics of Materials and Structures, University of Liege, Chemin des Chevreuils 1, 4000 Liege, Belgium
}

\begin{abstract}
This paper introduces a numerical 2.5D model of continuous casting of steel slabs. This model is based on the finite element method and it has been applied to the study of some local defects in a continuous caster, such as partial blockage of nozzles (leading to a local reduction of secondary cooling rate), locking or misalignment of rolls. The purpose of the study was the evaluation of the effect of such defects on the risk of transverse cracking during bending and unbending operations. To do so, the simulation at macro-scale of the complete process has been first performed in standard conditions to get reference values and then each defect has been introduced. Defining two indexes (indicators) of the risk of transverse cracking, it has been possible to classify the defects in terms of risk increase, helping steel producers to focus on the most critical problems.
\end{abstract}

Keywords: Continuous casting; Transverse crack; Nozzle blockage; Roll locking; Misalignment; Finite element

\section{PROCESS PRESENTATION AND CHALLENGES}

Continuous casting has progressively replaced ingot casting over the years 70s and 80s. Nowadays it is the most common way to produce cast steel thanks to its advantages: better quality, higher yield and lower costs (in manpower and energy). The process is schematically drawn in Fig. 1: it consists in pouring a mould with molten metal, i.e. steel in the present case, that starts solidifying in contact with the walls of the mould (primary cooling). When the process is launched, the bottom of the mould is a dummy that is moving down at a constant speed, so-called casting speed, allowing a constant supplying of the process. When the dummy reaches the exit of the mould, the solidified skin of the strand should be thick and resistant enough to avoid breaking. At this moment, the core of the strand is still liquid. To keep on cooling, the surface of the strand is then sprayed with water at each roll interval (secondary cooling), so that solidification front finally reaches the core of the strand, which can be cut and generally sent to next process (rolling). Once the process has been launched, the caster is continuously supplied in molten steel and the production does not stop until the geometry (cross section) of cast product must be changed. Obviously, this short presentation of continuous casting remains simplified and partial and it does not introduce all the complexity of such a process. For further reading, a lot of information is available in literature as well as on the internet.

The quality of continuously cast products is still increasing, thanks to the development and the improvement of the technique. To do so, many studies have been conducted by different teams. Among others, Brimacombe and co-workers are recognized to have helped going ahead in understanding the keys of the process, see [4$6,2,3,9,30]$. Meanwhile, the growth of computer's potential progressively enabled the advent of powerful numerical models. Thomas and co-workers at University of Illinois have also developed many applications in this field - for some of most recent publications, see [19,21,22,27,32,34,36].

Yet several challenges remain and crack prevention is one of the most important topics to reach the highest quality. The control of operating factors can be used to minimize such defects formation: the cooling rate and steel composition are the most obvious parameters. In [5], the different types of internal and surface cracks that can appear during continuous casting are examined. Two ranges of temperature correspond to low ductility in steels and these are identified as responsible for cracking:

- At very high temperature, microsegregation of solute elements at solidifying dendrites interfaces reduces ductility of steel in the semi-solid phase, i.e. at the solidification front. Therefore the strength and ductility of steel values are small in the temperature range close below the solidus temperature [6]. This loss of ductility is the origin and allows propagation of cracks along interden-drites (internal cracks) and probably account for surface longitudinal cracks.

- At a lower temperature, the $\gamma$-austenite- $\alpha$-ferrite phase transformation also reduces the hot ductility of steel in 
the temperature range usually between a lower limit $T_{1}$ (about $700-750^{\circ} \mathrm{C}$ ) and an upper limit $T_{2}\left(900-1100^{\circ} \mathrm{C}\right.$ ) depending on steel composition. This second low ductility zone is related to the appearance of surface transverse cracks.

Fig. 1: Sketch of continuous casting process.

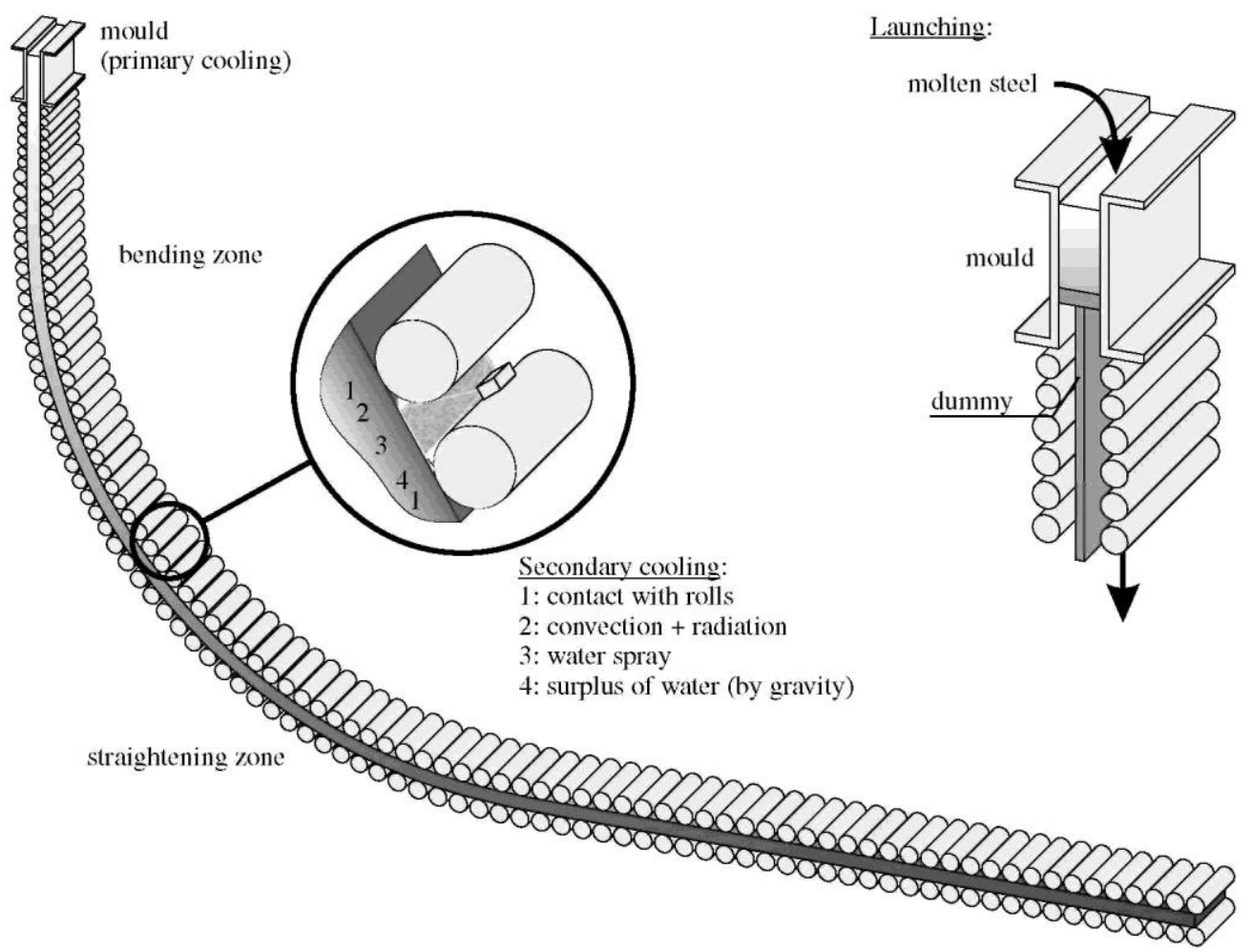

While other authors focused on the behavior of the strand in the mould [16], the present model focuses on (surface) transverse cracking, i.e. on the second range of temperature defined above and thus essentially on the secondary cooling since such temperature on the slab surface is reached in the lower part of the caster. Unfortunately, this zone also corresponds to the unbending of the slabs, that is to say where mechanical loadings are applied. The combination of such loadings and the loss of ductility is responsible for possible initiation of transverse cracks.

Besides these aspects, oscillation marks largely contribute to the mechanism. In [15], the link between segregation, low ductility, oscillation mark formation and transverse cracking is clearly underlined. Mintz $[23,24]$ realized a large review on hot ductility of steels and its relationship to the problem of transverse cracking in continuous casting. He demonstrated the importance of four variables that mostly control hot ductility: the strain rate, the grain size, the precipitation and inclusion contents.

The measures to prevent transverse cracking can be classified into two categories: control of the steel chemistry (which is essential) and prevention of excessive thermal and mechanical loadings during the process. This second topic was the principal objective of our study. To do so, two different approaches have been set up:

(1) a macroscopic approach, considering the material as a continuum, applying boundary conditions and determining the state variables at the macroscopic scale (stresses, strains, temperature, etc.);

(2) a mesoscopic approach modelling the material at the grain scale taking account for damage evolution in grain boundaries, the applied boundary conditions being the macroscopic thermomechanical loadings found in the first approach.

These two approaches are complementary. We present in this paper the first approach with some examples of application to the casting of a steel slab in standard conditions and in case of some local defects occurring in the unbending zone: reduction of water spray cooling, roll locking and roll misalignment. The purpose of the study was to evaluate the increase of risk of transverse cracking due to such defects using macroscopic indicators 
based on thermal state of the material (temperature) and mechanical loadings (longitudinal stress and strain). The description of the second approach (at mesoscale) can be found elsewhere [28,7,8].

\section{NUMERICAL MODEL}

\subsection{Constitutive equations}

Steel, like any current alloy, solidifies over a range of temperature depending on its composition, especially the carbon content. Such a kind of phase transformation is characterized by two temperatures: the liquidus temperature $T_{\text {liq }}$ (beginning of solidification) and the solidus temperature $T_{\text {sol }}$ (completion of solidification). Between these two temperatures the solid fraction $f_{\mathrm{s}}$ increases from zero to one. In a first approximation, we assumed a linear variation of the solid fraction $f_{\mathrm{s}}$ with respect to the temperature:

$0<f_{\mathrm{s}}(T)=\frac{T_{\text {liq }}-T}{T_{\text {liq }}-T_{\text {sol }}}<1 \quad \forall T \in\left[T_{\text {sol }} ; T_{\text {liq }}\right]$

Since solidification is an exothermic process, some energy (latent heat) is given off by the system and it must be taken into account in the balance of energy. The present model is based on fixed grid method, i.e. both solid and liquid are treated as one continuous material, with different properties, but without any explicit boundary from the mesh point of view [18]. Though the interval of phase transformation $T_{\text {liq }}-T_{\text {sol }}$ is relatively large according to temperature variations during a time step, the model integrates a general enthalpy method $[10,26,31]$ based the variation of enthalpy per unit mass function $\mathscr{H}$

$$
\mathscr{H}(T)=\int_{0}^{T}\left(c-L_{\mathrm{f}} \frac{\partial f_{\mathrm{s}}}{\partial \theta}\right) \mathrm{d} \theta,
$$

where $\rho$ is the density, $c$ the specific heat and $L_{\mathrm{f}}$ the latent heat of phase transformation (fusion-solidification) per unit mass. The main advantage of such a formulation is that the size of time step does not influence the result because the conservation of heat is always verified. The temperature field $T$ is then governed by the local expression of heat conservation

$\rho c \frac{\partial T}{\partial t}=\nabla \cdot(k \nabla T)+\dot{q}$

where $k$ is the thermal conductivity of the material. The parameter $\dot{q}$ is a heat source term that corresponds either to heat exchange (at the boundary of the domain), either to the latent heat $L_{\mathrm{f}}$ released during the phase transformation

$\dot{q}=\rho L_{\mathrm{f}} \frac{\partial f_{\mathrm{s}}}{\partial T} \dot{T}$.

The use of enthalpy function $\mathscr{H}$ leads to the well-known expression of heat conservation

$\dot{\mathscr{H}}(T)=\nabla \cdot(k \nabla T)$.

One can notice that all the material parameters $p, c, k$ and $\dot{q}$ are temperature dependent.

On the boundary $\delta \Omega$ of the studied domain, heat transfer coefficient $h_{\mathrm{tc}}$ governs heat extraction rate $\dot{q}$ :

$\dot{q}=h_{\mathrm{tc}}\left(T-\bar{T}_{\infty}\right)$,

where $\bar{T}_{\infty}$ is the external temperature (rolls, room or water). The heat transfer coefficient depends on the mode of heat exchange. The cooling of the steel slab is carried out thanks to devices spraying water on the slab surface. Four types of zone can be identified along the casting direction (repetitively, cf. Fig. 1):

(1) contact between the slab and the rolls, 
(2) heat loss by convection and radiation (to rolls and surroundings),

(3) sprayed zones,

(4) heat loss to surplus water in roll gap.

The industrial partner of the research provided all the $h_{\mathrm{tc}}$ coefficients. For the sprayed zones, these coefficients have been experimentally determined on hot steel specimens in a broad range of temperature (700-1200 $\left.{ }^{\circ} \mathrm{C}\right)$ for different water spray flows and for different models of nozzles. The same experiments allowed determining $h_{\mathrm{tc}}$ in convection-radiation zones thanks to measurements without spraying the specimens. Finally, a global $h_{\mathrm{tc}}$ coefficient for both contact zones and heat losses to water surplus had been previously calibrated using a 1D finite difference model and some temperature measurements at the surface of the slab (pyrometers).

The mechanical analysis involves the determination of stresses and strains development due to solidification, cooling of the strand and applied forces. The total strain rate tensor $\dot{\varepsilon}_{i j}$ is decomposed into mechanical and thermal contributions $\dot{\varepsilon}_{i j}^{\text {mech }}$ and $\dot{\varepsilon}_{i j}^{\text {th }}$. Since the model does not explicitly predict solid phase transformations, their contribution to the total strain rate must be implicitly expressed. An apparent thermal linear expansion coefficient $\alpha$ is used to model volume variations due to temperature variations and consequent phase transformations (from $\delta$-ferrite to $\gamma$-austenite and $\alpha$-ferrite).

The mechanical strain rate $\dot{\varepsilon}_{i j}^{\text {mech }}$ is composed of an elastic and a viscous-plastic parts. The elastic domain is modeled assuming a linear isotropic elastic material (Hooke's law)

$\sigma_{i j}=\hat{\sigma}_{i j}+\sigma_{\mathrm{m}} \delta_{i j}=2 G \hat{\varepsilon}_{i j}+3 \chi \varepsilon_{\mathrm{m}} \delta_{i j}$,

where $G$ is the shear modulus, $\chi$ the bulk modulus and $\delta_{i j}$ the Kronecker delta. In the above expression, the ${ }^{\wedge}$ symbol is used for deviatoric tensors and the ' $m$ ' subscript for mean values. Time integration of Hooke's law (7) takes account for linear variation of elastic properties during the time step

$$
\begin{aligned}
\sigma_{i j, B}= & \left(\frac{G_{B}}{G_{A}} \hat{\sigma}_{i j, A}+2 G_{B} \hat{\dot{\varepsilon}}_{i j} \Delta t\right) \\
& +\left(\frac{\chi_{B}}{\chi_{A}} \sigma_{\mathrm{m}, A}+3 \chi_{B} \dot{\varepsilon}_{\mathrm{m}} \Delta t\right) \delta_{i j}
\end{aligned}
$$

where $A$ and $B$ subscripts correspond to the value of the variables at the beginning and the end of time step $\Delta t$. In the viscous-plastic domain, the time integration of stress tensor $\sigma_{i j}$ is based on a plasticity flow rule associated to a von Mises' yield surface with isotropic hardening

$$
\hat{\dot{\varepsilon}}_{i j}=\frac{3 \overline{\dot{\varepsilon}}}{2 \bar{\sigma}} \hat{\sigma}_{i j}
$$

where $\bar{\sigma}=\sqrt{\frac{3}{2} \hat{\dot{\sigma}}_{i j} \hat{\dot{\sigma}}_{i j}}$ is the (von Mises) equivalent stress and $\overline{\dot{\varepsilon}}=\sqrt{\frac{2}{3} \dot{\varepsilon}_{i j} \dot{\varepsilon}_{i j}}$ is the equivalent strain rate.

The material constitutive law is a kind of Norton-Hoff law that has been modified to introduce softening. This is illustrated in Fig. 2, where the 'classical' Norton-Hoff formulation

$$
\bar{\sigma}=\sqrt{3} K(\sqrt{3} \overline{\dot{\varepsilon}})^{m} \bar{\varepsilon}^{n}
$$

cannot correctly fit to an experimental curve (tensile test on a steel specimen at $1000{ }^{\circ} \mathrm{C}$ and at a constant strain rate of $10^{-4} \mathrm{~s}^{-1}$ ). The following modified formulation is then proposed:

$\bar{\sigma}=\sqrt{3} K(\sqrt{3} \overline{\dot{\varepsilon}})^{m} \bar{\varepsilon}^{n} \mathrm{e}^{-p \bar{\varepsilon}}$

The parameters $K, m, n$ and $p$ are characteristics of the material and temperature dependent: 
- $m$ is the sensitivity of the stress to the strain rate $\dot{\varepsilon}$ and it can vary from 0 to 1 ;

- $n$ is the sensitivity to the strain level;

- $K$ is a proportionality factor (stiffness);

- $p$ is the parameter acting on the additional factor " $\mathrm{e}^{-p \bar{\varepsilon}}$ " that behaves like a decreasing exponential - the influence of this factor is larger in the large strains range, thus $p$ can be tuned to model softening of the material, i.e. decreasing of stress while strain increases.

All these parameters can be identified using uniaxial compression test. In the application presented hereafter they have been provided for the considered steel grade by the steel producer from its large data bank of parameters.

The relation between deviatoric stress and strain rate tensors, i.e. $\hat{\sigma}_{i j}$ and $\hat{\dot{\varepsilon}}_{i j}$ respectively, can be derived from (9) and (11)

$\hat{\sigma}_{i j}=2 K(\sqrt{3} \overline{\dot{\varepsilon}})^{m-1} \bar{\varepsilon}^{n} \mathrm{e}^{-p \bar{\varepsilon}} \hat{\dot{\varepsilon}}_{i j}$

The time integration of the constitutive law is based on an implicit scheme. The stress rate tensor $\dot{\sigma}_{i j}$ can be split into deviatoric $\hat{\dot{\sigma}}_{i j}$ and mean $\dot{\sigma}_{\mathrm{m}}$ parts

$$
\left\{\begin{array}{l}
\hat{\dot{\sigma}}_{i j}=2 G\left(\hat{\dot{\varepsilon}}_{i j}^{\text {mech }}-\hat{\dot{\varepsilon}}_{i j}^{\mathrm{vp}}\right)+\frac{1}{G} \frac{\partial G}{\partial T} \hat{\sigma}_{i j} \dot{T}=f_{i j}(T)+g_{i j}\left(\hat{\sigma}_{i j}, \bar{\varepsilon}, T\right), \\
\dot{\sigma}_{\mathrm{m}}=3 \chi\left(\dot{\varepsilon}_{\mathrm{m}}^{\mathrm{mech}}-\dot{\varepsilon}_{\mathrm{m}}^{\mathrm{vp}}\right)+\frac{1}{\chi} \frac{\partial \chi}{\partial T} \sigma_{\mathrm{m}} \dot{T}
\end{array}\right.
$$

If $\hat{\sigma}_{i j}^{A}$ is the deviatoric stress tensor at the beginning $A$ of the time step $\Delta t$, the value at the end $B$ of the step is given developing the relation (13) in Taylor series to the first order

$$
\begin{aligned}
\hat{\sigma}_{i j}^{B}= & \hat{\sigma}_{i j}^{A}+\left(f_{i j}^{A}(T)+g_{i j}^{A}\left(\hat{\sigma}_{i j}, \bar{\varepsilon}, T\right)\right) \Delta t \\
& +\left(\left.\frac{\partial f}{\partial T}\right|_{A} \theta \Delta T_{A B}\right) \Delta t+\left(\left.\frac{\partial g}{\partial \hat{\sigma}_{i j}}\right|_{A} \theta \Delta T_{A B}\right) \Delta t \\
& +\left(\left.\frac{\partial g}{\partial \bar{\varepsilon}}\right|_{A} \theta \Delta T_{A B}\right) \Delta t+\left(\left.\frac{\partial g}{\partial T}\right|_{A} \theta \Delta T_{A B}\right) \Delta t
\end{aligned}
$$

where $0<\theta<1$ is a time integration parameter (equal to $2 / 3$ in the model, according to Galerkin's time integration scheme).

In the same way, the time integration of mean stress $\sigma_{\mathrm{m}}$ is governed by

$$
\sigma_{\mathrm{m}}^{B}=\sigma_{\mathrm{m}}^{A}+\left(\frac{1}{\chi} \frac{\partial \chi}{\partial T} \sigma_{\mathrm{m}}^{A} \dot{T}\right) \Delta t+3 \chi\left(\dot{\varepsilon}_{\mathrm{m}}-\dot{\varepsilon}_{\mathrm{m}}^{\mathrm{th}}\right)
$$

To simplify the equations, the model is presented in small strain formalism in this paper. Obviously, large displacements, strains and rotations are correctly handled in the finite element code. Strains and stresses are computed incrementally at each integration point of the finite elements assuming a constant material velocity gradient $\dot{F}_{i j}$ over the time step $\Delta t$. Then, the Jaumann-Zaremba stress rate tensor $\stackrel{\circ}{\sigma}_{i j}$ is used to maintain objectivity of stress tensor in the context of large rotations

$$
\stackrel{\circ}{\sigma}_{i j}=\dot{\sigma}_{i j}-\omega_{i k} \sigma_{k j}+\sigma_{i k} \omega_{k j}
$$

where $\omega_{i j}=\frac{1}{2}\left(L_{i j}-L_{j i}\right)=-\omega_{j i}$ is the skew-symmetric part of the spatial velocity gradient $L_{i j}=\dot{F}_{i k} F_{k j}^{-1}$ or spin 
tensor.

Fig. 2: Comparison of an experimental curve, the 'classical' Norton-Hoff formulation (Eq. (10)), and the modified formulation including softening (Eq. (11)).

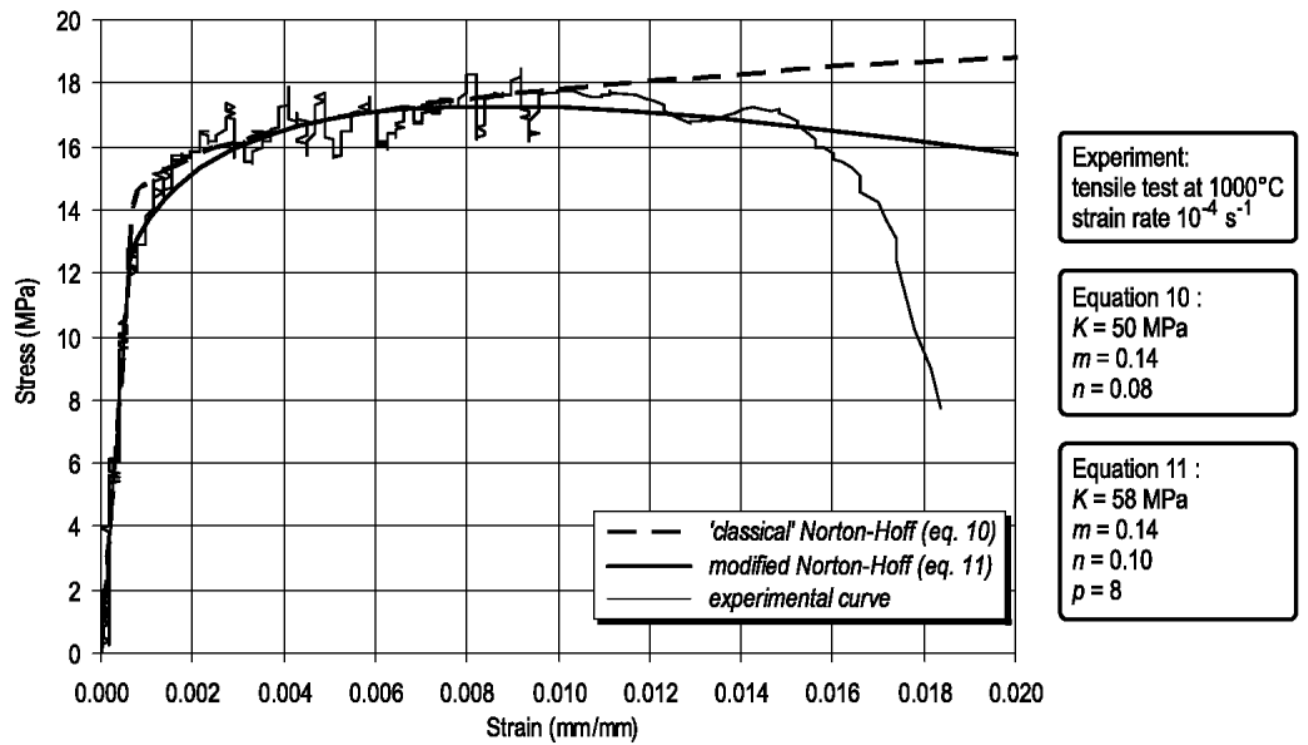

As long as molten steel is present in the core of the strand, the liquid applies a hydrostatic pressure on the solidified shell. This analogy with water loading leads to define this pressure as the ferrostatic pressure $p_{\mathrm{f}}$ : it is a hydrostatic-type stress and it is equal to the specific weight y of the liquid (molten steel) multiplied by the depth under free surface $D$, In the liquid phase, the ferrostatic pressure is fully developed, whereas in the solid phase it is obviously equal to zero. In the semi-solid zone, several assumptions could be discussed, but we assumed a linear variation of the pressure with respect to the liquid fraction $f_{1}=1-f_{\mathrm{s}}$. Since the solid fraction $f_{s}$ has been assumed to linearly vary with temperature, cf. relation (1), the ferrostatic pressure $p_{\mathrm{f}}$ is computed by

$p_{\mathrm{f}}= \begin{cases}\gamma D & \text { if } T_{\mathrm{liq}}<T, \\ \gamma D\left(\frac{T-T_{\mathrm{sol}}}{T_{\mathrm{liq}}-T_{\mathrm{sol}}}\right) & \text { if } T_{\mathrm{sol}}<T<T_{\mathrm{liq}}, \\ 0 & \text { if } T<T_{\mathrm{sol}} .\end{cases}$

A specific finite element has been introduced in the code for ferrostatic pressure loading. This element, designated as BOCHA element in our code, is an isoparametric 8-nodes Q2 two-dimensional element (quadratic and quadrangular) that is equivalent to 4 uni-dimensional loading elements applying a uniformly distributed loading on each of the four edges of the element - see Fig. 3. Each element is connected to the 8 nodes defining one plane element (so the geometry of both elements is and remains identical). To derive the ferrostatic pressure $p_{\mathrm{f}}$, the average temperature in the element is computed at its center and $p_{\mathrm{f}}$ is determined according to the relation (17) and assumed to be uniform in the element (only one integration point is necessary). The nodal forces are then computed and applied perpendicularly and outward the element as shown in Fig. 3.

Fig. 3: Isoparametric 8-nodes finite element for ferrostatic pressure loading (BOCHA element).

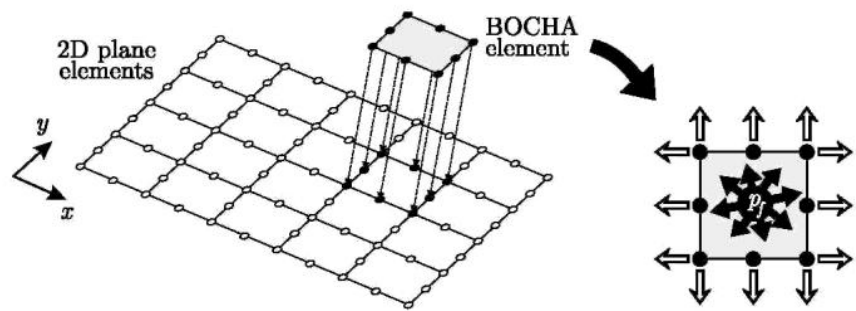




\subsection{Generalized plane strains formulation}

A complete three-dimensional discretization seemed unworkable because of numerical stability and convergence problems, but also CPU cost. A so-called "2.5D" approach has been preferred, taking advantage of the generalized plane strain formulation. This approach has also been adopted by Li [19] in CON2D continuous casting model. It consists in modelling a thin slice perpendicular to the casting direction and moving down through the caster. Assuming a constant casting speed, time scale becomes equivalent to the position of the slice in the caster. The slice is meshed using 2D 8-nodes isoparametric finite elements corresponding to the middle plane and the thickness $e$ anywhere in the slice is governed by the following relation:

$e(x, y)=\alpha_{0}+\alpha_{1} x+\alpha_{2} y$,

where appear 3 additional degrees of freedom $\alpha_{0}, \alpha_{1}$ and $\alpha_{2}$. Since these degrees of freedom are identical for all the mesh (not dependent on $x$ and $y$ coordinates), the thickness $e$ varies linearly and the slice is always confined between two planes, which are symmetric with respect to the middle plane of the slice. This formulation allows taking account for out-of-plane stresses $\sigma_{z z}$ and strains $\varepsilon_{\mathrm{zz}}$.

In other respects, generalized plane strains also permit to model bent slices: considering a slice in the bent zone of the caster as a slice situated between two planes converging to the center of curvature, the succession in time (thus along casting axis) of several bent slices reproduces the curvature of the steel strand, see Fig. 4 . Then, the geometry of the caster defines the relations between degrees of freedom $\alpha_{0}, \alpha_{1}$ and $\alpha_{2}$. Considering Fig. 5, local axes $x$ and $y$ are in the plane of the slice and $z$ is aligned on casting direction:

- $\alpha_{1}$ is equal to zero (no thickness variation along $x$ direction);

- $R$ is the radius of curvature of the strand (generally measured from the outer face);

- $h$ is the thickness of the slab (i.e. the width of the slice);

- the two planes confining the slice are converging in OO', the center axis of curvature, leading the following relation between $\alpha_{0}$ and $\alpha_{2}$.

$$
e_{\mathrm{OO}}=0=\alpha_{0}+\alpha_{2}\left(R-\frac{h}{2}\right)
$$

Fig. 4: Succession of slices in the bent zone.

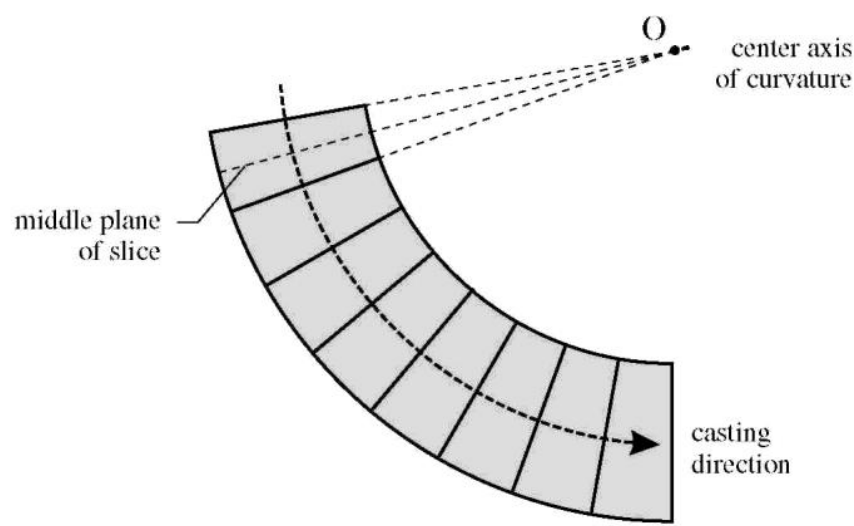

\subsection{Correction of bulging predicted by slice models}

Because of the ferrostatic pressure, the slab is bulging between successive rolls. Classical 2D slice models are not able predicting correct bulging because of the lack of shear stress in the out-of-plane direction: the absence of such a stress does not allow transmitting shear force, which is of prime importance for a correct modelling. The solidified shell behaves like a tube under pressure. At a first glance (and neglecting creep), the deflection is 
analogous to a beam clamped at both extremities and uniformly loaded - see Fig. 6a. In lack of transmission of shear stress, each slice (perpendicular to the casting direction) bulges independently of the behavior of the previous and the next slice, globally providing a bulging similar to the one presented in Fig. $6 \mathrm{~b}$.

To face up to this problem, an original approach is proposed by introducing in the model an external force to reproduce the effect of the missing shear stress. Consider the slab without rolls: it would bulge freely, like a tube under pressure. Each wide face would deform more or less like a plate clamped along two opposite edges and uniformly loaded - see Fig. 7. Assuming an elastic linear behavior for the plate, the deflection $w_{1}(\mathrm{x})$ of such a plate can be approached by

$$
w_{1}(x)=\frac{q L_{2}^{4}}{\pi^{4} \frac{E h^{3}}{1-v^{2}}} 1.5\left(1-\cos \frac{2 \pi x}{L_{2}}\right),
$$

where $q$ is the uniform loading, $E$ elastic modulus, v Poisson's coefficient, $h$ the thickness of the plate and $L_{2}$ the span of the plate between clamped edges (corresponding to slab width). This corresponds approximately to the bulging of the slab predicted by classical slice models (the shear stress $\tau_{y z}$ being equal to zero), except that the no-penetration of slab in the rolls would limit the bulging.

Actually, the bulging of the slab corresponds rather to the deflection of uniformly loaded plate clamped along its four edges. In such a case, shear stress $\tau_{y z}$ is not equal to zero. Assuming the same linear elastic behavior, the deflection $w_{2}(\mathrm{x}, z)$ of such a plate is approached by

$$
\begin{aligned}
w_{2}(x, z) & =\frac{q L_{2}^{4}}{\pi^{4} \frac{E h^{3}}{1-v^{2}}} \frac{\left(1-\cos \frac{2 \pi z}{L_{1}}\right)\left(1-\cos \frac{2 \pi x}{L_{2}}\right)}{\left(\frac{L_{2}}{L_{1}}\right)^{4}+\frac{2}{3}\left(\frac{L_{2}}{L_{1}}\right)^{2}+1} \\
= & \frac{q L_{2}^{4}}{\pi^{4} \frac{E h^{3}}{1-v^{2}}} \frac{\left(1-\cos \frac{2 \pi z}{L_{1}}\right)\left(1-\cos \frac{2 \pi x}{L_{2}}\right)}{\lambda^{4}+\frac{2}{3} \lambda^{2}+1},
\end{aligned}
$$

Fig. 5: Geometry of a bent slice.

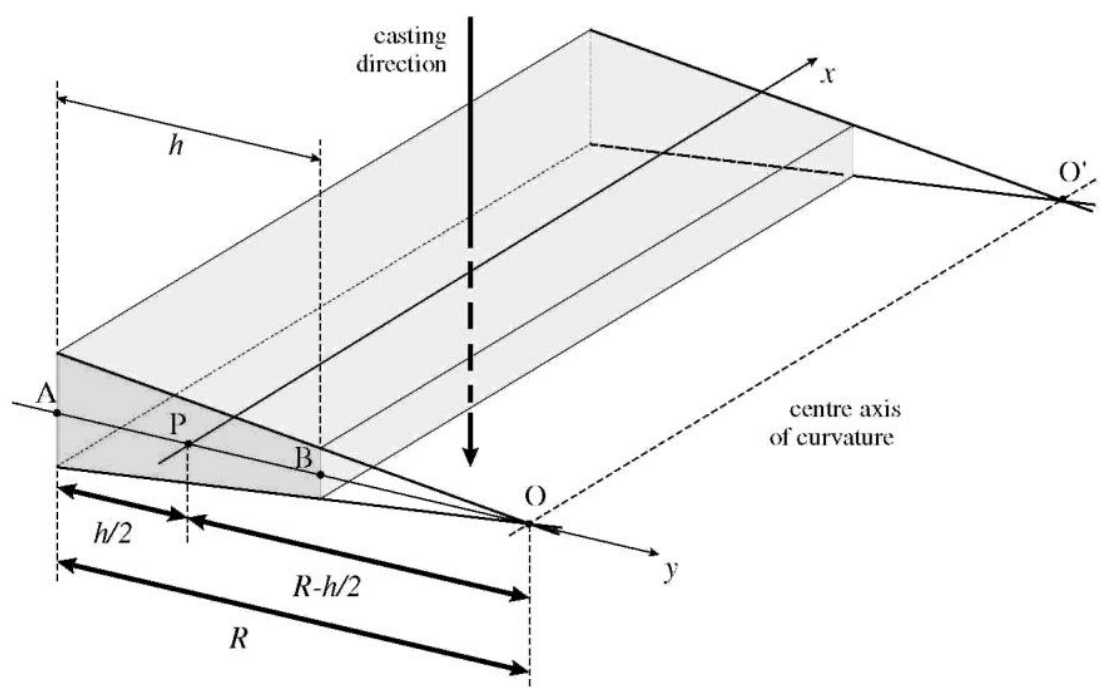


Fig. 6: To the left (a): Shear force transmission in a continuous strand under ferrostatic pressure (analogy with a beam clamped at both extremities and uniformly loaded). To the right (b): Bulging of the strand in lack of shear stress transmission between successive slices (classical 2D "slice" models).

a

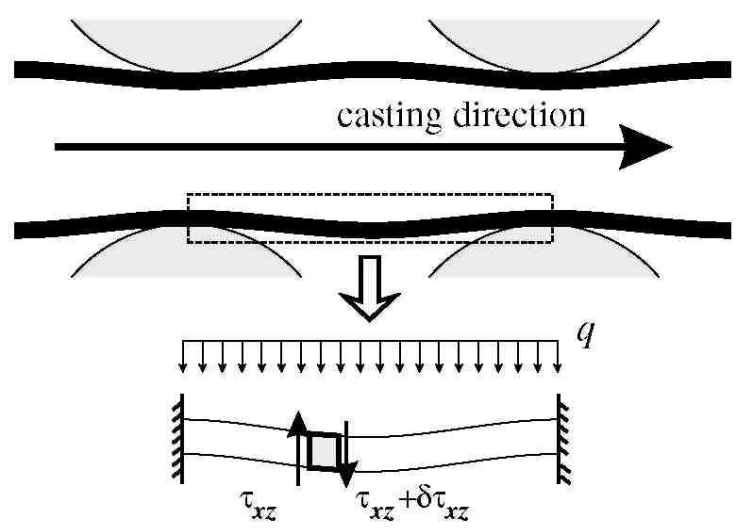

b

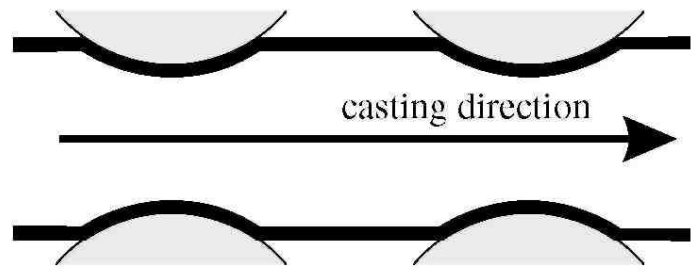

Fig. 7: Deflection of a uniformly loaded plate clamped along two/four edges.
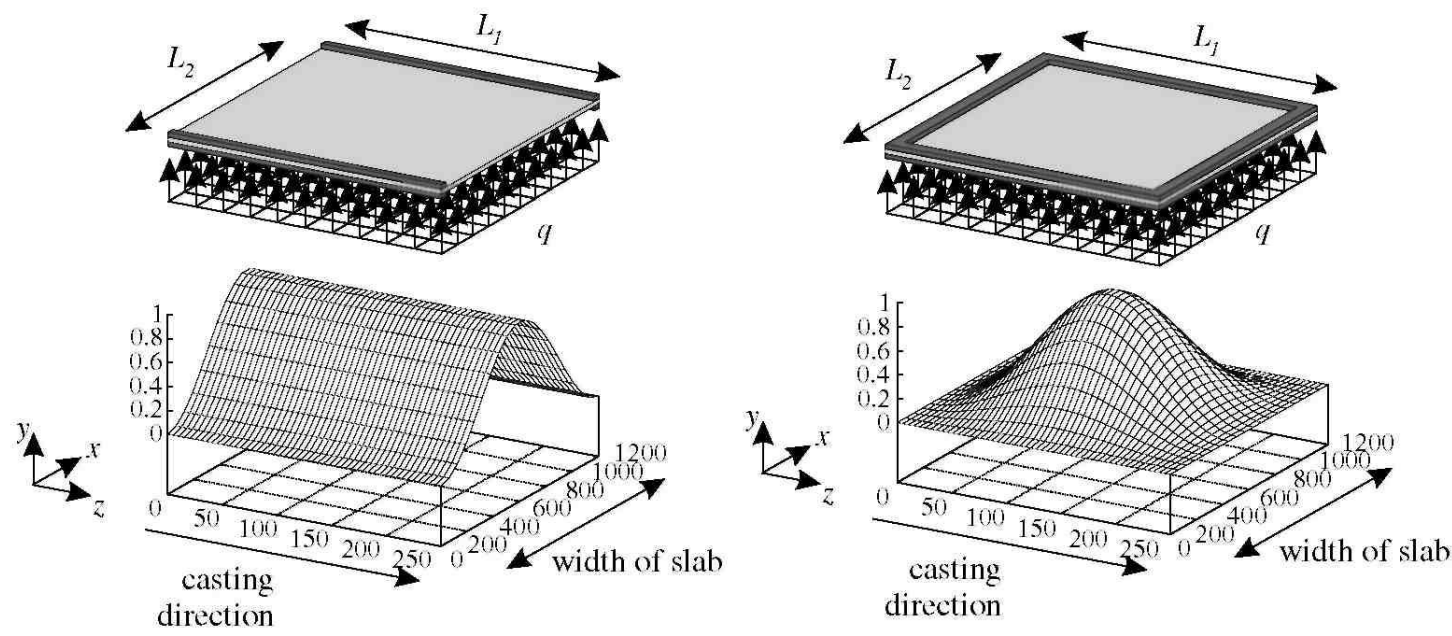

where $L_{1}$ and $L_{2}$ are spans along $\mathrm{z}$ - and $\mathrm{x}$-directions (respectively casting and transverse directions). To simplify notation, one can introduce $\lambda=\frac{L_{2}}{L_{1}}$ the aspect ration of the plate. If the bulging could follow such a shape, and taking into account the no-penetration condition, it would be something like Fig. 8.

A quick computation of maximum bulging with typical parameters in continuous casting can be operated with relations (20) and (22). Considering: $q=p_{\mathrm{f}}=0.2 \mathrm{~N} / \mathrm{mm}^{2}$ (equivalent to about $3 \mathrm{~m}$ of fluid pressure), $E=10 \mathrm{GPa}$ (corresponding to a temperature in the range of $1300-1400{ }^{\circ} \mathrm{C}$ ), $v=0.3, h=30 \mathrm{~mm}, L_{1}=250 \mathrm{~mm}$ and $L_{2}=$ $1200 \mathrm{~mm}$, the maximum deflections (bulging) would be $w_{1}\left(\frac{L_{2}}{2}\right)=40 \mathrm{~mm}$ and $w_{2}\left(\frac{L_{1}}{2}, \frac{L_{2}}{2}\right)=0.1 \mathrm{~mm}$.

This proves that if the shear stress is not taken into account, the maximum deflection can be several magnitudes larger than it should be (in the present case: 400 times).

To reduce bulging obtained by the slice model, it was therefore necessary to introduce an additional force, which could simulate the application of shear force. The evaluation of such a force is not trivial. However, the result is rather well known: the force should lead to obtain a bulging similar to the one defined by relation (22). To do so, springs have been introduced in the model, one at each node of the wide faces. The stiffness of the springs is added to the stiffness of the solidified shell, so that the bulging under ferrostatic pressure is reduced since the effort is partly balanced by the springs and the solidified shell (see Fig. 9).

Since the model remains a slice model, the bulging is given by relation (20). However, because of the introduction of springs, only a part of the ferrostatic pressure $p_{\mathrm{f}}$ is applied on the solidified shell, this part $q$ being 
equal to

$q=p_{\mathrm{f}}-f$,

where $f$ is the force in the springs (per unit surface). The applied force $q$ being reduced, the bulging $w_{1}(x)$ is also reduced and the higher the stiffness of the springs is, the lower the bulging is. Now considering that the stiffness of the springs is varying in time (so with respect to casting direction), the loading part $q$ also becomes a function of time, or $z$

$q(z)=p_{\mathrm{f}}-f(z)$

meaning that bulging also varies with respect to casting direction

$w_{1}(x, z)=\frac{q(z) L_{2}^{4}}{\pi^{4} \frac{E h^{3}}{1-v^{2}}} 1.5\left(1-\cos \frac{2 \pi x}{L_{2}}\right)$.

Fig. 8: Wide faces of a portion of slab bulging with rolls and shear stress.

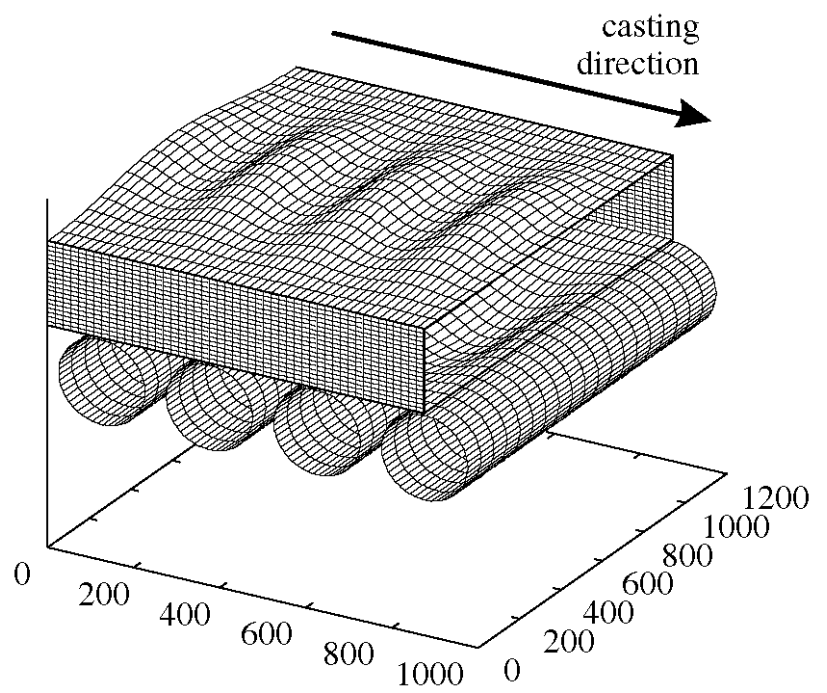

That way, the bulging has always the shape of a cosine curve, but the amplitude of the "wave" can be modified changing the stiffness of the springs. Now, to obtain a bulging equivalent to relation (22), the value of the pressure $q(z)$ can be computed thanks to the equation

$w_{1}(q, x, z)=w_{2}\left(p_{\mathrm{f}}, x, z\right)$

or

$$
\begin{gathered}
\frac{q(z) L_{2}^{4} 1.5\left(1-\cos \frac{2 \pi x}{L_{2}}\right)}{\pi^{4} \frac{E h^{3}}{1-v^{2}}}=\frac{p_{\mathrm{f}} L_{2}^{4}\left(1-\cos \frac{2 \pi z}{L_{1}}\right)\left(1-\cos \frac{2 \pi x}{L_{2}}\right)}{\pi^{4} \frac{E h^{3}}{1-v^{2}}\left[\lambda^{4}+\frac{2}{3} \lambda^{2}+1\right]} \\
\leftrightarrow \quad q(z)=\frac{p_{\mathrm{f}}}{1.5} \frac{1-\cos \frac{2 \pi z}{L_{1}}}{\lambda^{4}+\frac{2}{3} \lambda^{2}+1} .
\end{gathered}
$$

The relation (28) defines the load $q(z)$ that must be applied to the slice model in order to obtain a bulging equivalent to a 3D model, the bulging of which would be given by relation (22). To obtain such a load $q(z)$, the 
springs must balance an effort given by

$$
f(z)=p_{\mathrm{f}}-q(z)
$$

In other respect, the elongation of the springs $w_{3}$ is equal to the bulging of the slab

$$
w_{1}(q, x, z)=w_{2}\left(p_{\mathrm{f}}, x, z\right)=w_{3}(f, x, z)
$$

So the stiffness $k$ of the springs must be equal to

$$
\begin{aligned}
k(x, z) & =\frac{f(z)}{w_{3}(f, x, z)} \\
& =\left[1.5 \frac{\lambda^{4}+\frac{2}{3} \lambda^{2}+1}{1-\cos \frac{2 \pi z}{L_{1}}}-1\right] \frac{\pi^{4} \frac{E h^{3}}{1-v^{2}}}{1.5 L_{2}^{4}\left(1-\cos \frac{2 \pi x}{L_{2}}\right)} .
\end{aligned}
$$

Using such an approach, the maximum bulging does not exactly match the one observed in practice. In fact, it would be Utopian to believe that the bulging follows the equation of an elastic plate: the material is not elastic (but rather visco-plastic with creep effect), temperature (thus material behavior) is not uniform towards the shell thickness, and ferrostatic pressure is not constant from one roll to the next one. Therefore, it is not surprising to obtain bulging relatively different from the real one. However, the shape of bulging is much closer to the real one than with the classical 2D "slice" models (without springs). So the idea was to apply an amplification factor $\eta$ to the spring stiffness $k$ to fit the maximum bulging $w_{\max }$ on a reference value, provided by direct measurements on the caster or using a bulging model as those found in literature. Obviously, any other surface equation than relation (22) can be imposed in the model. Such a relation can be found using different kinds of model (analytical or empirical). Literature provides many examples of study on bulging in different casting conditions and according to different assumptions [12,25,20,11,17,29,13,35].

In the application presented here, maximum bulging has been computed using a model developed by IORC (Industrial Operation Research Center of ARCELOR Group -formerly IRSID). This model should be considered as a black box, the input data being: the ferrostatic pressure, the roll pitch (distance between two successive contacts with the rolls), the thickness of the solidified shell, the surface temperature, the Young's modulus, the consistency and the sensibility to strain and strain rate of the material. The output of the model is the maximum bulging $\mathrm{W}_{\text {max }, \mathrm{i}}$, for each roll interval $i$ along the caster: the interval number $i$ is between rolls number $i-1$ and $i$, the first interval being between the exit of the mould and the first pair of rolls. The results of IORC model are presented in Fig. 10 and they are compared to the results obtained with the LAGAMINE model $(2.5 \mathrm{D}$ model + elastic springs + amplification factor $\left.\eta_{i}\right)$.

Fig. 9: Reduction of slice bulging under ferrostatic pressure thanks to springs.
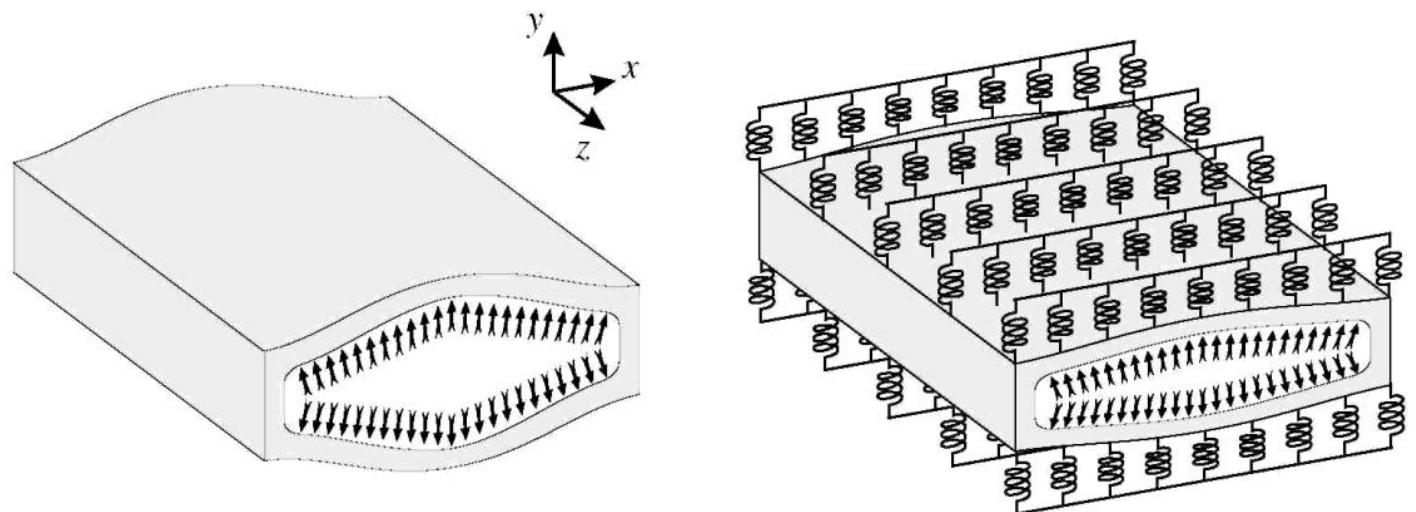
Fig. 10: Maximum bulging of the slab vs. number of interval between rolls - comparison between IORC model and Lagamine model.

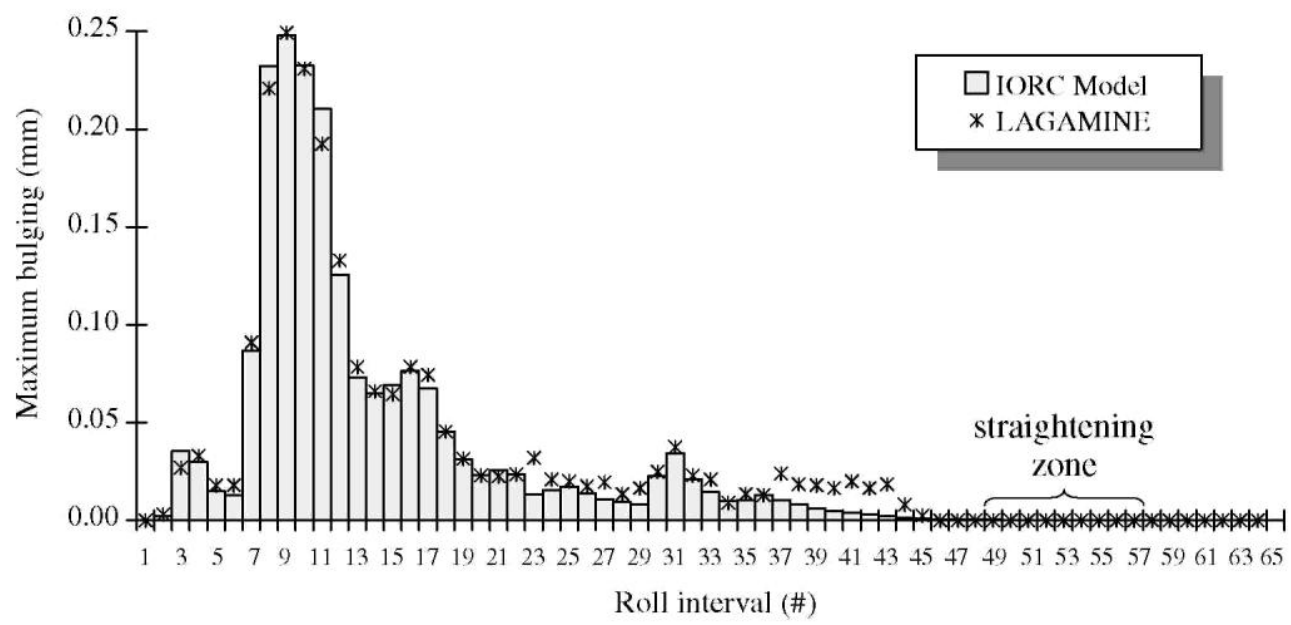

\subsection{Evaluation of extraction force}

Due to ferrostatic pressure, the strand inflates between successive pairs of rolls, applying on the rolls an effort $\delta F$. To maintain a constant velocity of the strand in the caster (casting speed), it is necessary to apply an extracting force $\delta R$ to balance the frictional resistance equal to

$$
\delta R=\mu_{\mathrm{axis}} \delta F
$$

where $\mu_{\text {axis }}$ is a dry friction coefficient for the rolls spinning around their axis. In other respect, bending and unbending of the strand requires applying additional force and resting on the supporting rolls, so that the effort $\delta F$ is higher in that zones where the curvature varies.

From a practical point of view, integrating contact pressure (between the strand and the roll) and multiplying by friction coefficient $\mu_{\text {axis }}$ would give the resistance $\delta R$. The existence of some measurements (conversion of electrical power into mechanical work) allows having an idea of the applied extraction force. Such measurements have been performed on the studied caster in standard casting conditions for a $220 \mathrm{~mm}$ x $1200 \mathrm{~mm}$ slab (see Fig. 11).

From roll to roll, the resistance is rising so that the slab is in tensile state along casting direction (effective longitudinal stress $\sigma_{z z}$ perpendicularly to the slice). Some pairs of rolls are motorized (extracting rolls): they are distributed along the caster at different places and they are able to transmit an extraction effort by friction. The total applied effort must balance the resistance so that the progression of the slab in the caster can be guaranteed. In the present caster, more than a dozen of pairs of rolls are motorized. The repartition of the effort between the rolls is also subject to assumptions, each motorized roll not necessarily performing the same work. The measurements can be used in two different ways: either they are used for validation of the integration of friction (what we could call coupled approach, since extraction force is coupled to the contact computation), either they are input data (uncoupled approach).

The introduction of springs (cf. Section 2.3) strongly modifies the contact pressure, which tends to zero. The difficulty to determine the exact contact zone where friction must be integrated led us to consider the curve of Fig. 11 as an input data of the problem (uncoupled approach). In such a case, the effective longitudinal effort is an imposed force applied perpendicularly to the slice and it is balanced by the distribution of out-of-plane stresses $\sigma_{z z}$. The application of such an external force together with geometrical constrains (i.e. bending) is only possible thanks to the generalized plane strain approach. 


\subsection{Staggered time-stepping algorithm}

Casting process involves many complex phenomena and different kinds of coupling between thermal, mechanical and metallurgical aspects. Modelling such complexity at once cannot be achieved with present computers, hence the necessity to select the key phenomena to take account for according to the expected prediction: shell behavior, solidification, fluid flow [33]. In the present model, we only focus on the thermal state and mechanical loading on the solidifying shell. A simultaneous time-stepping algorithm was already implemented in the LAGAMINE code [14], as well as coupled thermo-mechanical constitutive laws. For reasons of numerical stability and computing time, the coupled problem has been split into two sub-problems: one governing the thermal problem and a second one the mechanical problem. A staggered time-stepping algorithm has been introduced to keep a weak coupling. This method is common in modelling of solidification processes and it leads to a stable scheme [1].

Fig. 11: Illustration of effective longitudinal effort in the slab and evaluation of the extraction force for a motorized pair of rolls.

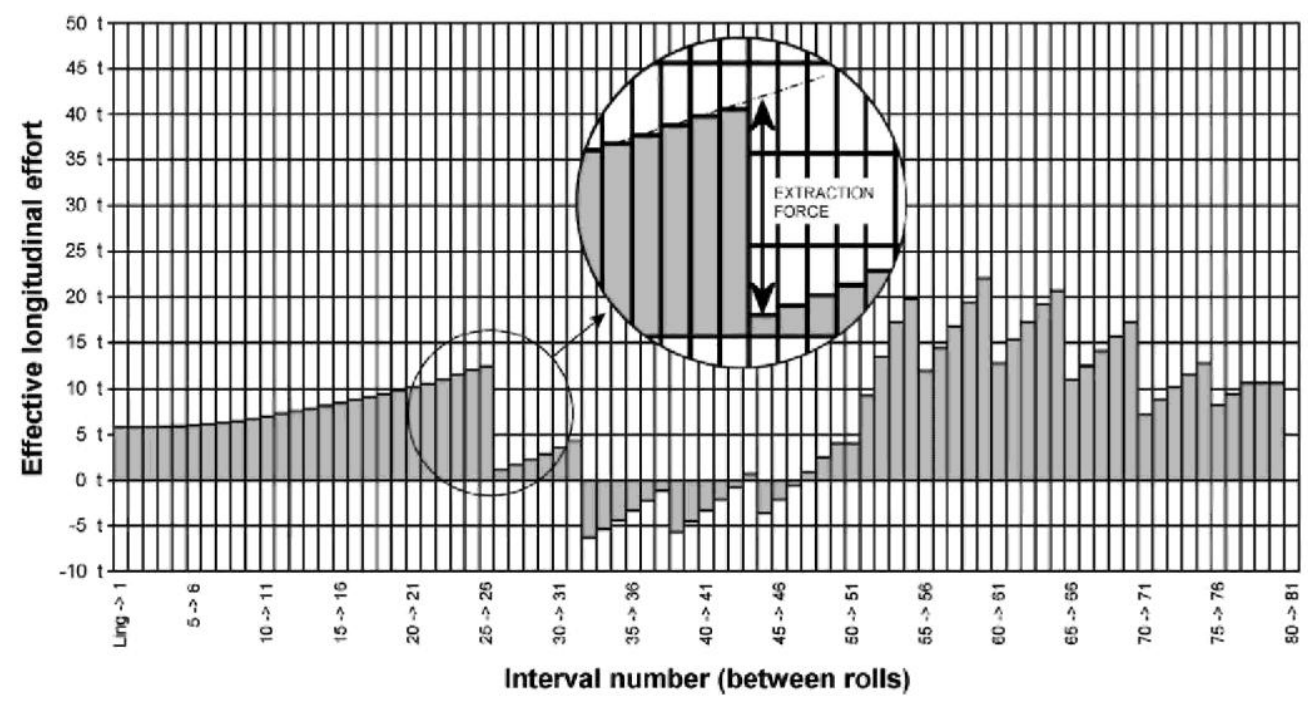

Fig. 12: Staggered time-stepping algorithm implemented in the present model.
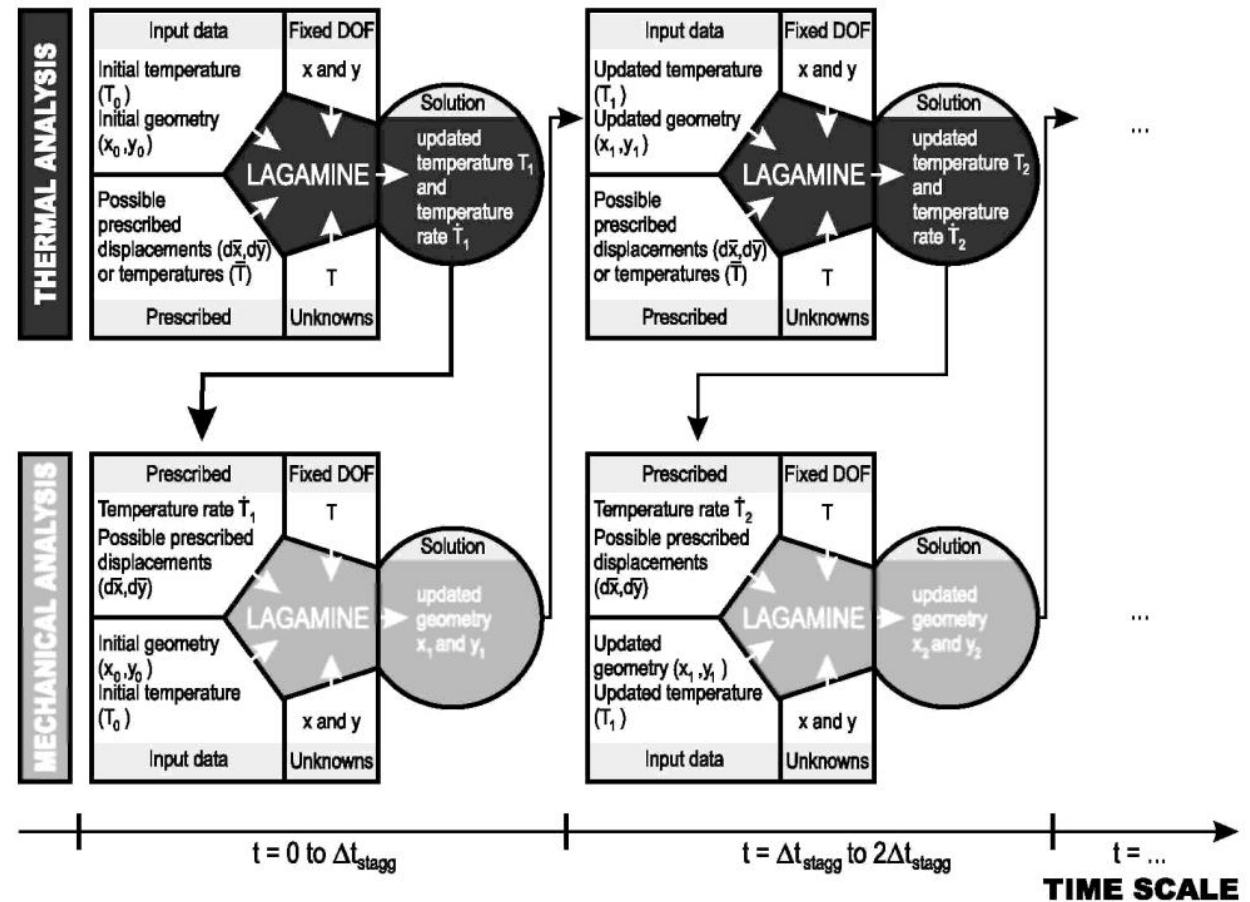
The staggered time-stepping scheme is described in Fig. 12. The two sub-problems are evolving in separate analyzes with different time steps. They are solved separately, each system of equations being easier to solve, since coupling terms between displacements and temperature evolution are not taken into account. The time scale is divided into intervals $\Delta t_{\text {stagg }}$ greater or equal to the minimum time step $\Delta t_{\text {min,th }}$ required for the thermal analysis to avoid oscillation and divergence of the solution.

During each time interval $\Delta t_{\text {stagg }}$, the thermal evolution is first computed assuming that all the nodes are fixed. The solution $T$ is then prescribed as data in the mechanical analysis on the same time interval $\Delta t_{\text {stagg, }}$, the nodes displacements being the unknowns this turn. For the next time intervals, the geometry of the thermal analysis is updated according to the mechanical analysis of the previous time interval and so on. In the application presented hereafter, $\Delta t_{\text {stagg }}=0.25 \mathrm{~S}$.

\subsection{Evaluation of the risk of transverse cracking}

Transverse cracking in continuous casting is caused by longitudinal tensile strains. Such strains are generated in the spray chambers or by the straightening. The latter one appears to result in crack formation if the surface temperature is too low, particularly in the range of $700-900{ }^{\circ} \mathrm{C}$ corresponding to the low temperature zone of low ductility $[5,23,24]$.

In order to evaluate the risk of transverse cracking, two simple indexes have been introduced. They are not explicit criteria in the sense that they do not explicitly predict crack initiation since no critical value is available (for example, yield stress could have been used to normalize indexes, if available). However, the value of indexes increases with the risk of transverse cracking so that it is possible to have a qualitative indication on the mechanical state of the slab in different situations. According to the objective of the study, the model only focuses on the surface transverse cracks, which generally appear in the corners. The conclusions of the studies of Brimacombe and Mintz naturally lead to the simple and intuitive definition of indexes based on both longitudinal stress $\sigma_{z z}$ and longitudinal strain rate $\dot{\varepsilon}_{z z}$, that have been identified as key factors in transverse crack formation. In addition, the two indexes combine also the metallurgical unfavorable aspects, i.e. the lack of ductility in the range of temperature between $T_{1}=700^{\circ} \mathrm{C}$ and $T_{2}=900^{\circ} \mathrm{C}$. They are established on the following bases:

- indexes are positives if mechanical state is favorable to open transverse cracks, equal to zero in other cases;

- indexes are significant when the material is characterized by low potential to deformation without cracking, i.e. when the ductility of the material is low;

- the higher the indexes are, the higher the risk is.

According to these conditions, the two following indexes $I_{1}$ and $I_{2}$ have been defined:

$$
\begin{aligned}
& I_{1}= \begin{cases}\max \left(0, \sigma_{z z}\right) & \text { if } T_{1}<T<T_{2}, \\
0 & \text { otherwise, }\end{cases} \\
& I_{2}= \begin{cases}\max \left(0, \dot{\varepsilon}_{z z}\right) & \text { if } T_{1}<T<T_{2}, \\
0 & \text { otherwise }\end{cases}
\end{aligned}
$$

\section{STUDY OF THE DIFFERENT DEFECT CASES}

\subsection{Definition of the local defects}

Three types of local defect have been considered:

- The first defect is a partial blockage of nozzles leading to a reduction of the water spray rate and thus a lower heat extraction. We assumed that the defect concerns one of the circuit, located in the unbending zone (between rolls number 47 and rolls number 52). The water rate has been reduced to $66 \%$ of the nominal value.

- The second defect is the locking of either one, either two pairs of rolls. In a first time we considered one locked pair in the constant curvature zone (pair number 48 -just before unbending zone) and in a second case, we considered a second locked pair in the unbending zone (number 52). 
- The third and last type of local defect is the misalignment of one pair of rolls. We chose the pair number 48 so that the effect of the misalignment can be easily compared to the effect of locking. The amplitude of the misalignment is a displacement of the pair equal to $2 \mathrm{~mm}$ towards the center of curvature of the caster.

For the first defect, only the heat transfer coefficients $h_{\mathrm{tc}}$ have been modified with respect to the standard conditions. One can note that the relationship between water rate and $h_{\mathrm{tc}}$ is not linear, but it must be correctly evaluated before to be introduced in the model.

For the second defect, we had to modify the extraction force applied on the strand. Since the rolls are locked, the strand is sliding on the rolls. The friction coefficient at these contact points is higher, increasing the resistance and thus requiring a higher extraction force. Assuming that $\delta R$ is the resistance in standard conditions (free rolls), the locking generates a higher resistance $\delta R^{*}$ that we assumed to be equal to

$\delta R^{*}=\frac{\mu_{\text {locked }}}{\mu_{\text {free }}} \delta R$

where $\mu_{\text {locked }}$ and $\mu_{\text {free }}$ are dry friction coefficients respectively in the case of locking and free rolls. For $\mu_{\text {locked }}$ we introduced a value equal to 0.30 and for $\mu_{\text {free }}$ a value equal to 0.015 (ball bearing), so that the locking of the pair of rolls multiplies the standard resistance $\delta R$ by a factor 20 .

For the third defect, the misalignment creates local successive positive and negative bending (see Fig. 13) and therefore additional resistance to be balanced by an increase of extraction force. In that case, we first used the coupled approach to evaluate the impact of local bending on the resistance by an amplification factor $\kappa_{i}$ for each pair of roll $i$, then we used the uncoupled approach with an amplified applied extraction force.

Fig.13: Successive local positive and negative bending due to misalignment.

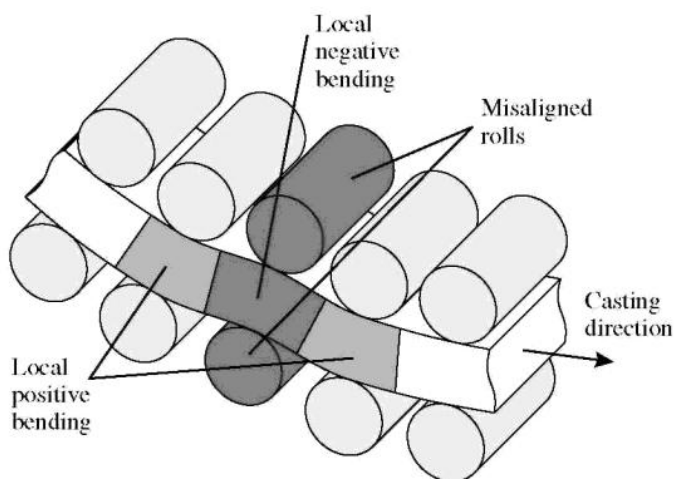

Table 1: Summary of the effect of local defects

\begin{tabular}{llll}
\hline Simulation & Max. value $\boldsymbol{I}_{\mathbf{1}}$ (MPa) & Max. value $\boldsymbol{I}_{\mathbf{2}}\left(\mathbf{s}^{-\mathbf{1}}\right)$ & Risk of cracking \\
\hline Standard conditions & 41.1 & $22 \times 10^{-5}$ & - \\
$\begin{array}{l}\text { Partial blockage } \\
\# 47 \rightarrow 52\end{array}$ & 41.1 & $22 \times 10^{-5}$ & Identical \\
Locking \#48 & 41.3 & $22 \times 10^{-5}$ & Almost identical \\
Locking \#48 \& 52 & 43.0 & $30 \times 10^{-5}$ & $\begin{array}{l}\text { Significant increase } \\
\text { Misalignment \#48 }\end{array}$ \\
& 46.0 (upper face) & $\begin{array}{l}40 \times 10^{-5} \\
\text { (second local positive } \\
\text { bending) }\end{array}$ & Large increase \\
& & & \\
\hline
\end{tabular}


Published in : Computer Methods in Applied Mechanics \& Engineering (2007), vol. 196, pp. 2285-5599

Status: Postprint (Author's version)

Fig. 14: First index $I_{1}$ in standard conditions (no local defect).

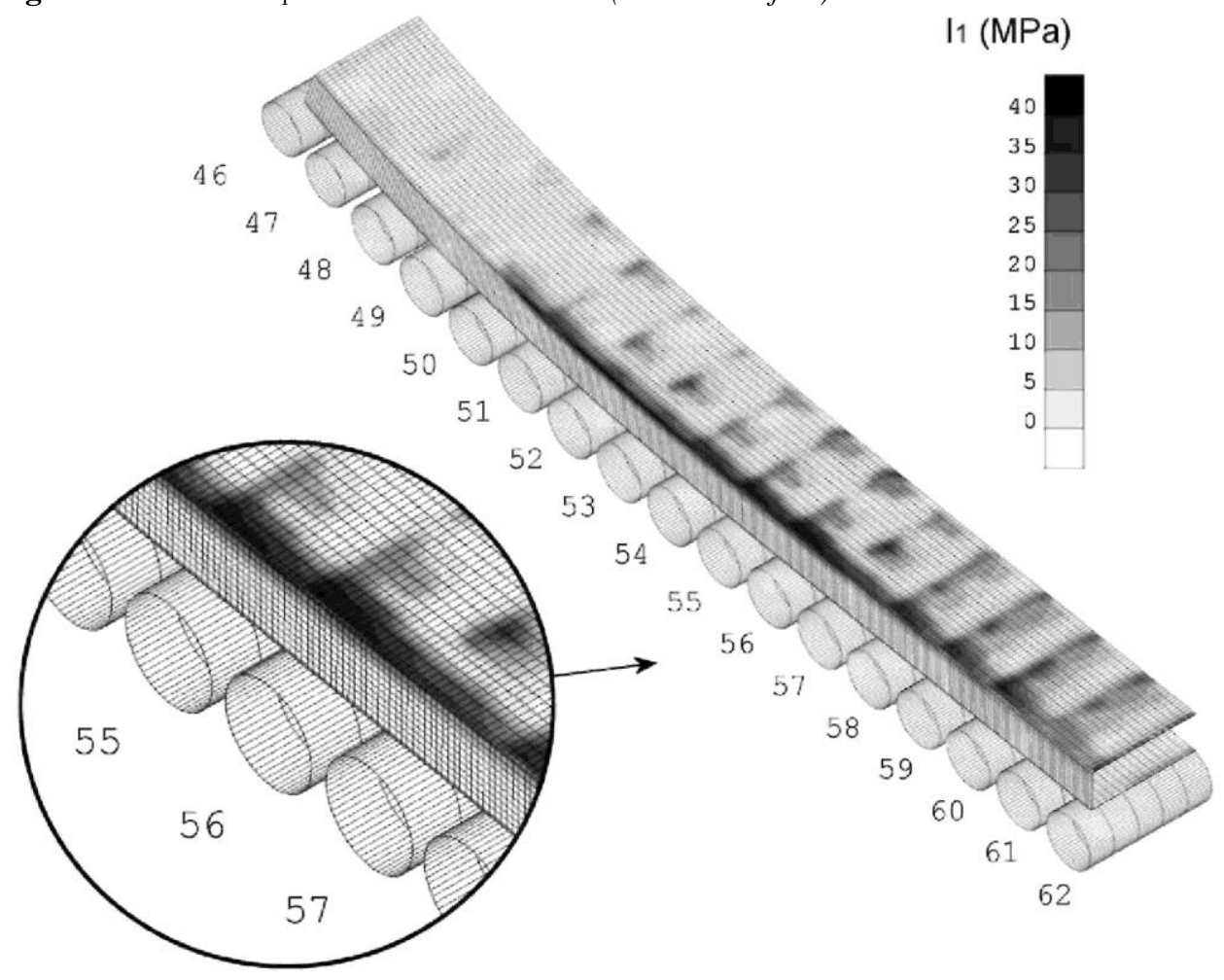

Fig. 15: Second index $I_{2}$ in standard conditions (no local defect).

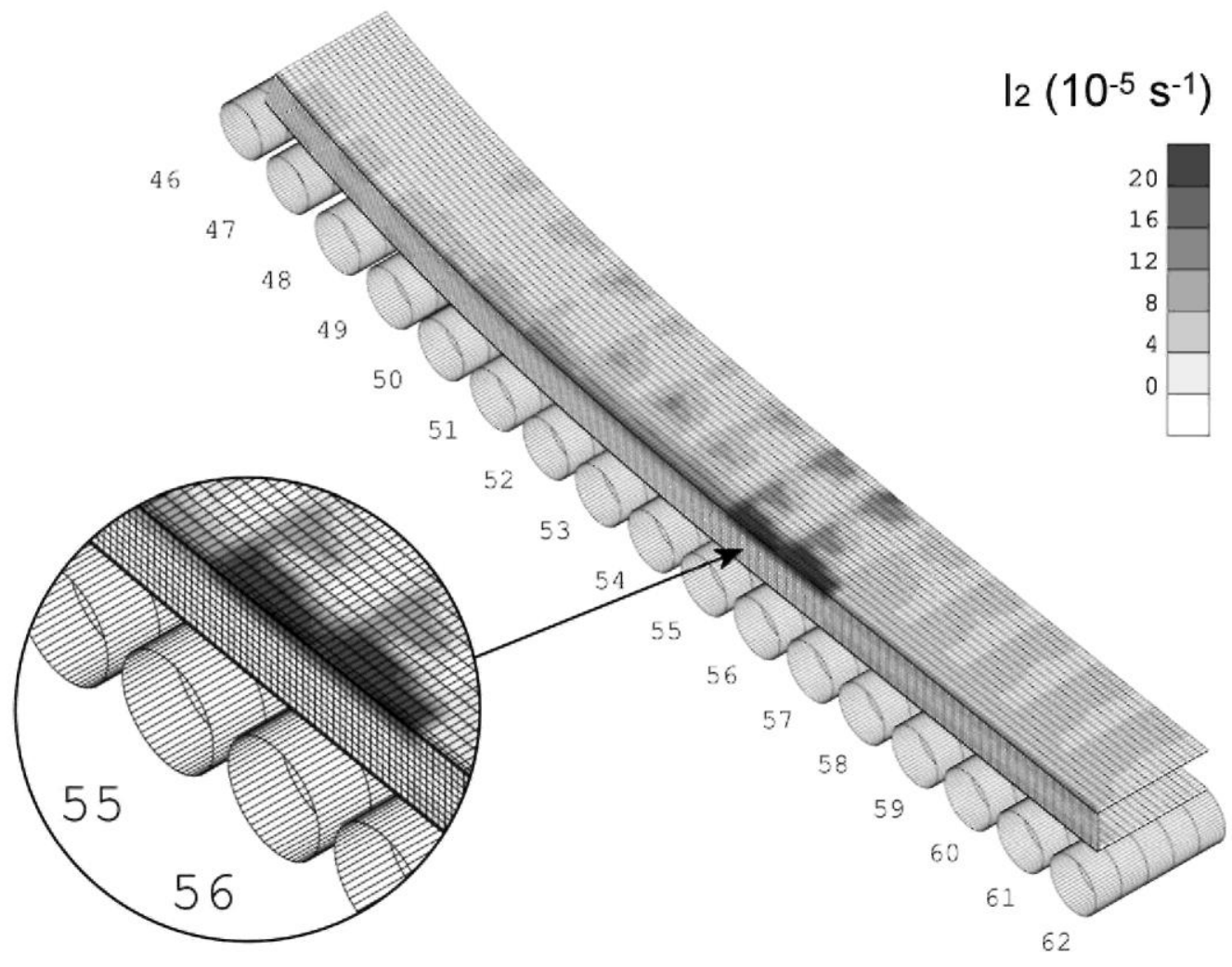




\subsection{Numerical results}

Fig. 14 represents the value of the first index $I_{1}$ under standard conditions (no local defect). This is a 3D mapping of the results: one half of the slab is studied because of the vertical symmetry plane and only the lower part is represented because from the top of the caster until the unbending zone, the surface temperature is generally higher than $T_{2}$ so that the indexes are equal to zero. The unbending zone begins at the pair of rolls number 49 and ends at pair number 57. It has been found that the most critical zone under standard conditions is the upper corner of the slab between rolls number 55 and 57, i.e. at the end of unbending. This conclusion matches the observations on steel slabs: when transversal cracks appear, they are generally observed near the corner on the upper face of the slabs. The second index $I_{2}$ shows similar indications (see Fig. 15).

The effect of each local defect on both indexes is summarized in Table 1. Comparing the maximum values of $I_{1}$ and $I_{2}$ in each case with respect to the standard conditions, one can classify the defects in terms of impact on the risk of transverse cracking (according to the increase of $I_{1}$ and $I_{2}$ ).

The analysis of the above results demonstrates that the reduction of the water cooling due to partial blockage does not modify the maximum risk level with respect to standard conditions. In fact, a lower cooling causes the strand core to reheat the surface and the temperature reaches higher values, sometimes above the upper limit temperature $T_{2}$ and thus reducing the area of critical zones. However, this positive effect is limited in time and affects only the central part of the slab surface, where the nozzles are spraying water. In the corner, the effect is not relevant and therefore maximum values of indexes $I_{1}$ and $I_{2}$ are still the same as in the standard case and located in the same area (end of unbending, on the corner of the upper face of the slab).

The locking of rolls seems to causes an increase of the risk of transverse cracking, according to the observed higher values of both indexes. In the case of rolls located in the constant curvature zone (\#48), the increase is slight, but in the case of a pair of rolls located in the unbending zone (\#52), the effect is much stronger. This is due to the fact that during unbending, the rolls are used as supports and the efforts applied are much larger than the single effect of ferrostatic pressure. The locking of such a pair generates a higher resistance that must be balanced by a higher extraction force, causing higher tensile state along casting direction. The most critical area remains the same, but the indexes are higher, indicating a higher risk of transverse cracking.

Fig. 16: Second index $I_{2}$ in case of rolls misalignment.

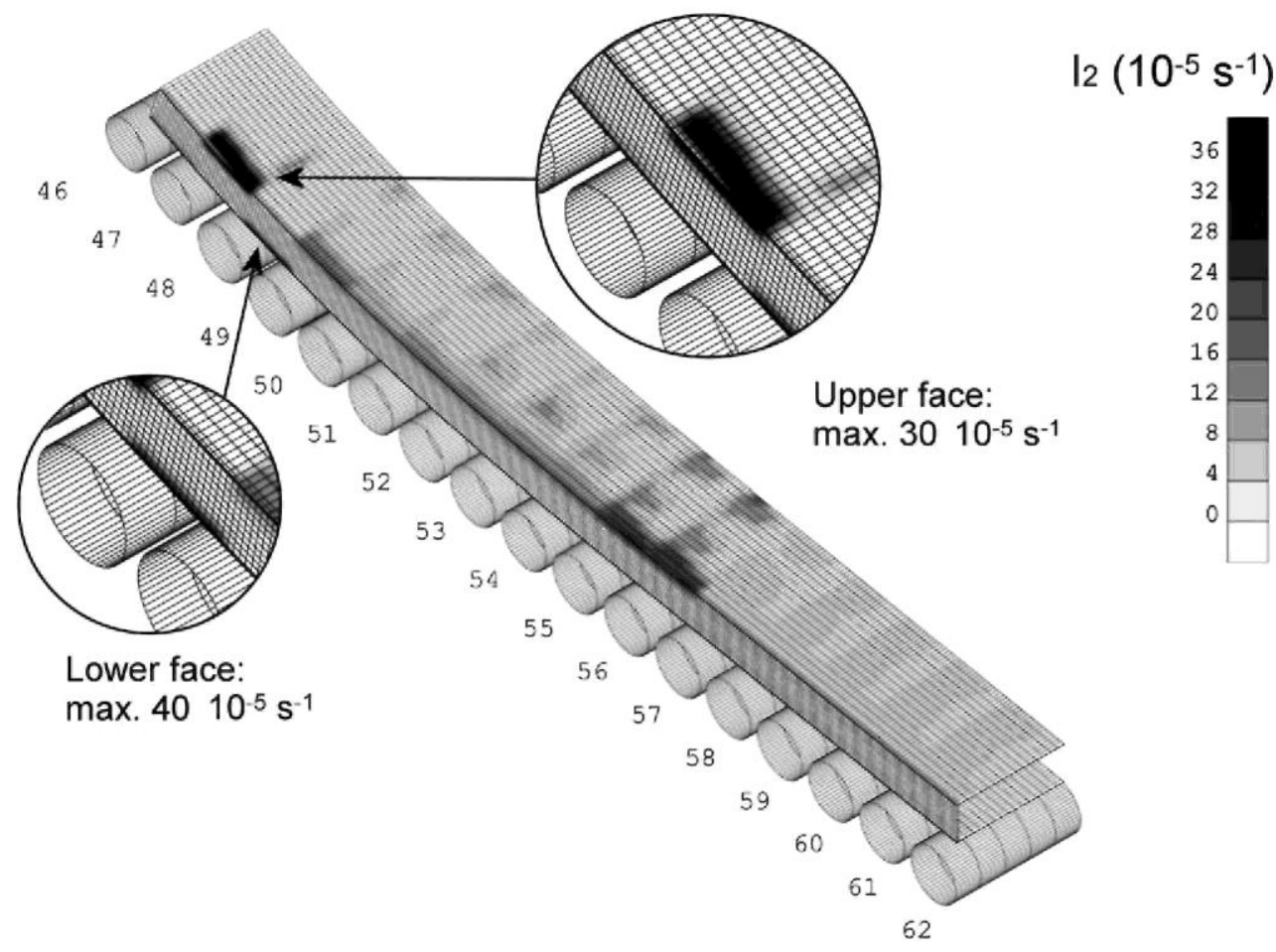

In case of misalignment, local positive and negative bending generate high longitudinal strain rates $\dot{\varepsilon}_{z \mathrm{z}}$. The maximum value of both indexes are much higher: $49.5 \mathrm{MPa}$ for $I_{1}$ and $40 \times 10^{-5} \mathrm{~s}^{-1}$ for $I_{2}$. These maximum values 
are located in the zone of the defect (misalignment), cf. Fig. 16. Highest values are found on corner of the lower face, because the second local positive bending is the most critical operation. The risk of transverse cracking in this case is the highest found among all the simulations.

\section{DISCUSSION AND CONCLUSIONS}

The presented model of continuous casting has been applied to steel slab casting in different cases: standard conditions and casting with some local defects occurring. The two indexes of risk of transverse cracking correctly indicate the most critical areas. In standard conditions, it has been found that the maximum risk occurs on the upper face, near the corner, at the end of unbending. This is in agreement with observation in practice (Fig. 14).

Comparing the indexes values, the model allows classifying different kinds of local defects in terms of increase in risk of transverse cracking. Three types of defects are presented: partial blockage of nozzles (reducing heat transfer rate), locking of rolls and misalignment of rolls. The conclusions and the classification obviously depend on the amplitude of the introduced defects. According to the ones used in this study, it has been found that the partial blockage of nozzles does not increase the risk of cracking, while the locking of rolls seems to have a higher impact on that risk, especially if the locked rolls are located in the unbending zone, i.e. when the supporting force applied one the locked rolls is larger. Concerning the misalignment of rolls, it has been found as the most critical studied defect, locally generating high values of longitudinal stress and strain rate (cf. indexes $I_{1}$ and $I_{2}$ ). However, it largely depends on the amplitude of the misalignment: in this case, $2 \mathrm{~mm}$ is a quite large value. In other respects, misalignment generally concerns not only one pair of rolls, but a complete segment of the caster, i.e. five or six consecutive pairs. Anyway, the presented model remains suitable for other analyzes of local defects, providing very explicit results that can be used easily to evaluate the impact such defects on the risk of transverse cracking. It constitutes a helpful tool for industries to determine priorities in caster maintenance.

\section{Acknowledgements}

The authors express their gratitude to the supporting industrial partner, ARCELOR, more especially the Technical Direction of Cockerill-Sambre (formerly CDAM) and the Industrial Operations Research Center (IORC, formerly IRSID). A.M. Habraken (as Research Director) and F. Pas-con (as Postdoctoral Researcher) also thank the National Fund for Scientific Research (Belgium) for its support.

\section{References}

[1] C. Agelet de Saracibar, A. Cervera, M. Chiumenti, On the formulation of coupled thermoplastic problems with phase-change, Int. J. Plast. 15 (1999) $1-34$

[2] LA. Bakshi, J.L. Brendzy, N. Walker, S. Chandra, I.V. Samarase- kera, J.K. Brimacombe, Mould-strand interaction in continuous casting of steel billets. Part 1. Industrial trials, Ironmak. Steelmak. 20 (1) (1993) 54-62.

[3] J.L. Brendzy, LA. Bakshi, I.V. Samarasekera, J.K. Brimacombe, Mould-strand interaction in continuous casting of steel billets. Part 2 . Lubrication and oscillation mark formation, Ironmak. Steelmak. 20 (1) (1993) 63-74.

[4] J.K. Brimacombe, Design of continuous casting machines based on a heat-flow analysis: state-of-the-art review, Can. Metall. Quart. 15 (2) (1976) 163-175

[5] J.K. Brimacombe, K. Sorimachi, Crack formation in the continuous casting of steel, Metall. Trans. B 8 (1977) 489-505.

[6] J.K. Brimacombe, I.V. Samarasekera, Principles of solidification and materials processing, in: R. Trivedi (Ed.), Proc. Indo-US Workshop, vol. 1, Trans Tech Publications, New Delhi, 1990, p. 179.

[7] S. Castagne, M. Remy, A.M. Habraken, Development of a mesoscopic cell modeling the damage process in steel at elevated temperature, Key Engrg. Mater. 223-236 (2003) 145-150.

[8] S. Castagne, F. Pascon, G. Blès, A.M. Habraken, Developments in finite element simulations of continuous casting, J. Phys. IV France 120 (2004) 447-455.

[9] S. Chandra, J.K. Brimacombe, IV. Samarasekera, Mould-strand interaction in continuous casting of steel billets. Part 3. Mould heat transfer and taper, Ironmak. Steelmak. 20 (2) (1993) 104-112. 
Published in : Computer Methods in Applied Mechanics \& Engineering (2007), vol. 196, pp. 2285-5599

Status: Postprint (Author's version)

[10] G. Comini, S. Del Guiduce, R.W. Lewis, O.C. Zienkiewicz, Finite element solution of non-linear heat conduction problems with special reference to phase change, Int. J. Numer. Methods Engrg. 8 (1974) 613-624.

[11] J.B. Dalin, J.L. Chenot, Finite element computation of bulging in continuously cast steel with a viscoplastic model, Int. J. Numer. Methods Engrg. 25 (1988) 147-163.

[12] A. Grill, K. Schwerdtfeger, Finite element of bulging produced by creep in continuously cast steel slabs, Ironmak. Steelmak. 6 (3) (1979) 131-135.

[13] J.S. Ha, J.R. Cho, B.Y. Lee, M.Y. Ha, Numerical analysis of secondary cooling and bulging in the continuous casting of slabs, J. Mater. Process. Technol. 113 (1-3) (2001) 257-261.

[14] A.M. Habraken, M. Bourdouxhe, Coupled thermo-mechanical-met- allurgical analysis during the cooling process of steel pieces, Eur.J. Mech. A - Solids 11 (3) (1992) 381-402.

[15] S. Harada, S. Tanaka, H. Misumi, S. Mizoguchi, H. Hiroguchi, A formation mechanism of transverse cracks on CC slab surface, ISIJ Int. 30(4) (1990) 310-316.

[16] A.E. Huespe, A. Cardona, V. Fachinotti, Thermomechanical model of a continuous casting process, Comput. Methods Appl. Mech Engrg. 182 (3-4) (2000) 439-455.

[17] N. Kanai, S. Horioka, M. Uehera, H. Saiki, Three-dimensional slab deformation of solidifying shell at intermediate bearing during high speed continuous casting, in: Proc. 80th Steelmaking Conf, Iron \& Steel Society, Warrendale, PA, USA, 1997, pp. 223-225.

[18] R.W. Lewis, K. Ravindran, Finite element simulation of metal casting, Int. J. Numer. Methods Engrg. 47 (1-3) (2000) 29-59.

[19] C. Li, B.G Thomas, Thermomechanical finite-element model of shell behavior in continuous casting of steel, Metall. Mater. Trans. B 35 (6) (2004) 1151-1172.

[20] S. Maeno, K. Wada, Y. Itoh, Y. Nagano, Measurement of bulging (studies on bulging of continuously cast slabs), Trans. ISIJ 24 (1984) B-82-B-83.

[21] Y. Meng, B.G. Thomas, Heat transfer and solidification model of continuous slab casting: CON1D, Metall. Mater. Trans. B 34 (5) (2003) 685-705.

[22] Y. Meng, B.G. Thomas, Modeling transient slag layer phenomena in the shell/mold gap in continuous casting of steel, Metall. Mater. Trans. B 34 (5) (2003) 707-725.

[23] B. Mintz, S. Yue, J.J. Jonas, Hot ductility of steels and its relationship to the problem of transverse cracking during continuous casting, Int. Mater. Rev. 36 (5) (1991) 187-217.

[24] B. Mintz, The influence of composition on the hot ductility of steels and to the problem of transverse cracking, ISIJ Int. 39 (9) (1999) $833-855$

[25] K. Miyazawa, K. Schwerdtfeger, Computation of bulging of continuously cast slabs with simple bending theory, Ironmak. Steelmak. 6 (2) (1979) 68-74

[26] K. Morgan, R.W. Lewis, O.C. Zienkiewicz, An improved algorithm for heat conduction problems with phase change, Int. J. Numer Methods Engrg. 12 (1978) 1191-1195.

[27] J.K. Park, B.G. Thomas, I.V. Samarasekera, Analysis of thermo- mechanical behavior in billet casting with different mold corner radii, Ironmak. Steelmak. 29 (5) (2002) 359-375.

[28] M. Remy, S. Castagne, A.M. Habraken, Progress in microscopic modeling of damage in steel at high temperature, Int. J. Forming Process. 5 (2-4) (2002) 445-455.

[29] J. Ren, Z. Wang, Analysis of bulging and strain for continuously cast slab as thermoelastic continuous beam, Ironmak. Steelmak. 25 (5) (1998) 394-397.

[30] I.V. Samarasekera, J.K. Brimacombe, Evolution or revolution? A new era in billet casting, Can. Metall. Quart. 38 (5) (1999) 347-362.

[31] C.R. Swaminathan, V.R. Voiler, A general enthalpy method for modeling solidification processes, Metall. Trans. B 23 (1992) 651-664

[32] B.G. Thomas, L. Zhang, Mathematical modeling of fluid flow in continuous casting: a review, ISIJ Int. 41 (10) (2001) $1185-1197$.

[33] B.G. Thomas, Continuous casting, in: Yu. Kuang-Oscar (Ed.), Modeling for Casting and Solidification Processing, Marcel Dekker Inc., New York, 2002, pp. 499-539. 
Published in : Computer Methods in Applied Mechanics \& Engineering (2007), vol. 196, pp. 2285-5599

Status: Postprint (Author's version)

[34] B.G. Thomas, M. Jenkins, R.B. Mahapatra, Investigation of strand surface defects using mold instrumentation and modelling, Ironmak. Steelmak. 31 (2004) 485-494.

[35] S. Toyoshima, M. Gotoh, K. Nakayama, Computation of three-dimensional deformation behavior of continuously cast steel strand in multiroll spans (parts 1 \& 2), J. JSTP 43 (502) (2002) 29-38.

[36] B. Zhao, S.P. Vanka, B.G. Thomas, Numerical study of flow and heat transfer in a molten flux layer, Int. J. Heat Fluid Fl. 26 (1) (2005) $105-118$ 